The Astrophysical Journal, 239:880-881, 1980 August 1

(C) 1980. The American Astronomical Society. All rights reserved. Printed in U.S.A.

\title{
POTENTIAL IN THE CENTRAL BAR STRUCTURE
}

\author{
Victor Szebehely, ${ }^{1}$ John Lundberg, and William J. McGahee \\ The University of Texas, Austin \\ Received 1979 August 3; accepted 1980 February 4
}

\begin{abstract}
The figure-eight orbits obtained by Miller and Smith inside the central bar structure of galaxies are used to establish possible potential functions which result in such orbits. It is shown that $r^{-6}$ type potentials are special cases of distance and angle-dependent potential functions.

Subject headings: galaxies: internal motions — galaxies: structure — stars: stellar dynamics
\end{abstract}

\section{INTRODUCTION}

In a recent paper Miller and Smith (1979) computed stellar orbits in a prolate bar and found that "about $25 \%$ of all orbits show clean or phase-shifted figure eights." The period of rotation of the bar is about 6 times larger than the period of the orbit; therefore, in the first approximation the rotation of the system may be neglected. This is especially true in the $(z, x)$-plane where the orbits show remarkable likeness to lemniscates (see Fig. 5 of Miller and Smith 1979, where the $x$ axis is along the bar and the body rotation axis is along $z$ ). If we consider motion only in the $(z, x)$-plane, neglect the rotation of the system, and assume "clean" figure-eight orbits, an analytical solution may be obtained for the potential function which results in such orbits.

It should be noted that in the $(y, x)$-plane Miller and Smith (1979) show that the centrifugal effects distort the clean figure eights and asymmetric loops appear. Our method is two-dimensional and sidereal (applicable in a fixed system); therefore, only motion in the $(z, x)$-plane is analyzed. Extension of our method to more general cases present fundamental analytical difficulties. Treatment in the synodic frame requires the solution of a nonlinear partial differential equation, while in the fixed system the pertinent partial differential equation is linear allowing an explicit analytic description of the general solution.

\section{FORMULATION OF THE INVERSE PROBLEM}

The inverse problem of dynamical astronomy may be defined as follows. For a given family of orbits find the force field which results in the orbits. It was shown before (Whittaker 1904) that this problem in general has no unique solution. In fact when conservative force fields are assumed the solution for the potential is obtained from a first-order linear partial differential equation (Szebehely 1974). Modifications and examples are offered by Broucke and Lass (1977) and by Morrison (1977).

This paper presents the general solution of the above

${ }^{1}$ L. B. Meaders Professor of Engineering. mentioned partial differential equation for figure-eight orbits or lemniscates given by the equation

$$
r^{2}=a^{2} \cos n \theta,
$$

where $r$ and $\theta$ are polar coordinates, $a$ is a scale factor, and $n$ is related to the angle $(\alpha)$ of the tangent to the lemniscate at the origin $(\alpha= \pm \pi / 2 n)$. Figure 1 shows a family of lemniscates with $a=1$ and $n=1,2,3$, and 4 . The figure-eight orbits of Miller and Smith (1979) correspond to $n>2$.

The partial differential equation which gives the potential function $(V)$ for a given orbit $f(x, y)=c$ may be written as (Szebehely 1974)

$\frac{2(V+h)}{f_{x}^{2}+f_{y}^{2}}\left(f_{x x} f_{y}^{2}-2 f_{x y} f_{x} f_{y}+f_{y y} f_{x}^{2}\right)+V_{x} f_{x}+V_{y} f_{y}=0$,

where $h$ is the constant of energy or the total energy per unit mass of the body moving in the field given by $V(x, y)$. Subscripts denote partial derivatives.

The same equation in polar coordinates becomes

$$
\begin{aligned}
\frac{2(V+h)}{f_{\theta}^{2}+r^{2} f_{r}^{2}}\left(f_{r r} f_{\theta}^{2}-2 f_{r} f_{\theta} f_{r \theta}\right. & \left.+f_{\theta \theta} f_{r}^{2}+r f_{r}^{3}+\frac{2}{r} f_{r} f_{\theta}^{2}\right) \\
& +V_{r} f_{r}+\frac{f_{\theta}}{r^{2}} V_{\theta}=0,
\end{aligned}
$$

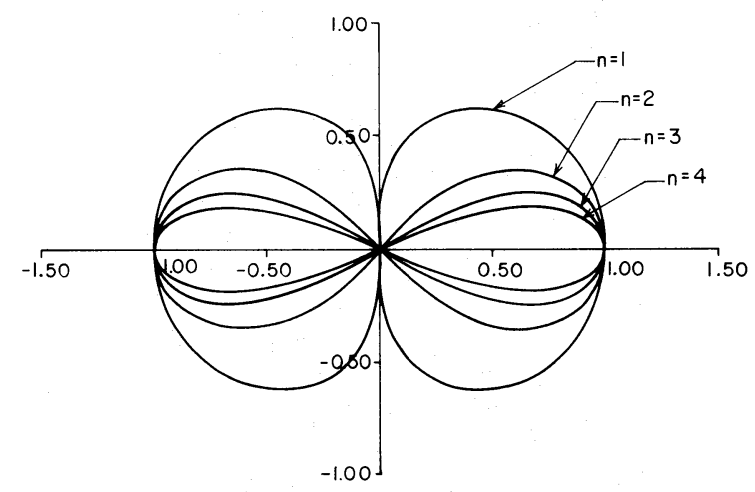

FIG. 1.-Family of lemniscates for $a=1$ and $n=1,2,3,4$ 
where the orbit is given by $f(r, \theta)=c$ and the potential function is $V(r, \theta)$.

\section{SOLUTIONS FOR THE POTENTIAL}

Substituting $r^{2}-a^{2} \cos n \theta=f(r, \theta)$ in equation (3), the partial differential equation for the determination of the potential becomes

where

$$
4(V+h) \phi+2 r V_{r}+n V_{\theta} \tan n \theta=0,
$$

$$
\phi=\frac{r^{4}\left(4-n^{2}\right)+3 n^{2} a^{4}}{r^{4}\left(4-n^{2}\right)+n^{2} a^{4}}
$$

The general solution of this equation may be obtained by the method of characteristics using

$$
\frac{d r}{2 r}=\frac{d \theta}{n \tan n \theta}=-\frac{d V}{4 \phi(V+h)} .
$$

From this system, the solution of equation (4) becomes

$$
V+h=\frac{a^{2} r^{4}\left(4-n^{2}\right)+n^{2} a^{6}}{r^{6}} F\left(\frac{a s^{2 / n^{2}}}{r}\right),
$$

where $s=\sin n \theta$ and $F$ is an arbitrary function of its argument. Since $a$ is a scale factor, we may normalize the result by considering $r / a$ instead of $r$ or simply using $a=1$. Note that the signs of the two terms in the numerator are opposite when $n>2$, i.e., for Miller and Smith (1979) type orbits.

\section{SPECIAL CASES}

1. When $F(x)=1$, we have

$$
V+h=\frac{n^{2}}{r^{6}}+\frac{4-n^{2}}{r^{2}} \text {. }
$$

From this we may conclude that a reduction in the potential (as well as in the radial force) occurs when $n \geq 2$. The near-field effects $\left(n^{2} / r^{6}\right)$ and the far-field effects $\left[\left(4-n^{2}\right) / r^{2}\right]$ operate in the same direction for broad $(n=1)$ lemniscates. But the forces are in the opposite direction for flat lemniscates when $n$ is large, as is the case for the figure eights of Miller and Smith (1979).

2. When $F(x)=x$, the potential function becomes

$$
V+h=\frac{r^{4}\left(4-n^{2}\right)+n^{2}}{r^{7}}(\sin n \theta)^{2 / n^{2}},
$$

which shows angular dependence as well as dependence on $r$.

3. The Newtonian gravitational component appears when $F(x)=x^{-1}$ is selected. This field is complicated with singularities occurring at the extremes of the loops of the lemniscates $(\theta=0, \pi / n)$, in addition to the singularities at the origin. The potential in this case is

$$
V+h=\left(\frac{4-n^{2}}{r}+\frac{n^{2}}{r^{5}}\right)(\sin n \theta)^{-2 / n^{2}} .
$$

The Newtonian potential term $\left(r^{-1}\right)$ is present as long as $n \neq 2$.

A number of other possibilities may be studied concerning equation (6) which is not a unique solution (as expected since it is the solution of a partial differential equation), and it contains an arbitrary function. In fact equation (4) may also be formulated in several different ways, which naturally influences the solution. Nevertheless, the approach is rather straightforward, and the results are not uninteresting.

Partial support from the National Science Foundation and from NASA are gratefully acknowledged. The first author is the L. B. Meaders Professor at the University of Texas, and assistance received from this professorship in the preparation of this paper is acknowledged. Comments received from Drs. $R$. Miller and P. Vandervoort (University of Chicago) and from Dr. R. Broucke (University of Texas) were most helpful in the preparation of this paper.

\section{REFERENCES}

Broucke, R., and Lass, H. 1977, Celestial Mechanics, 16, 215.

Miller, R., and Smith, B. F. 1979, Ap. J., 227, 785.

Morrison, R. 1977, Celestial Mechanics, 16, 227.

Szebehely, V. 1974, in Proc. Internat. Meeting on the Earth's
Rotation, ed. E. Proverbio (Bologna: University of Cagliari). Whittaker, E. T. 1904, Analytical Dynamics (Cambridge: Cambridge University Press).

John Lundberg, William J. McGahee, and Victor Szebehely: The University of Texas, Aerospace Engineering and Engineering Mechanics, WRW 217, Austin, TX 78712 